\title{
Comparaison de différentes méthodes de mesure de l'évapotranspiration d'une culture de blé non irriguée
}

\author{
J.M. Peterschmitt et N. Katerji \\ INRA, Station de Bioclimatologie, 78850 Thiverval-Grignon, France
}

(reçu le 29-4-1988, accepté le 4-12-1988)

Résumé - Durant 74 jours, représentatifs de l'ensemble du cycle végétatif d'une culture de blé non irriguée, nous comparons les évapotranspirations mesurées par évapotranspiromètre et celles calculées par la méthode du rapport de Bowen et par la méthode aérodynamique simplifiée. Les données de base utilisées sont celles obtenues par Paltridge et al. $(1972,1975)$ à Rutherglen (Australie) durant 2 années.

Les résultats indiquent un bon accord entre les méthodes aériennes et l'évapotranspiromètre. La méthode du rapport de Bowen est celle qui conduit aux valeurs les plus proches de l'évapotranspiromètre.

La comparaison entre la méthode du rapport de Bowen et la méthode aérodynamique simplifiée indique une légère tendance à la surestimation de l'évapotranspiration par la méthode aérodynamique simplifiée lorsque les flux de chaleur sensible sont importants.

Nous montrons enfin qu'une expression simple fonction du rayonnement net, du flux de chaleur dans le sol et de la température de l'air permet d'obtenir de bonnes estimations de l'évapotranspiration en fonction d'un coefficient cultural.

méthode du rapport de Bowen - méthode aérodynamique simplifiée - coefficient cultural

Summary - Comparison of different methods of measurement of evapotranspiration of non-irrigated wheat. On the basis of measurements made on non-irrigated wheat in Rutherglen, Australia (1971-1972) by Paltridge et al. (1972, 1975), we compare during a 74 day period including all phenological stages, actual evapotranspiration measured with a weighing lysimeter with values calculated by mean of two micrometeorological methods: the Bowen ratio method and the simplified aerodynamic method, proposed by Itier (1981).

The results show a good agreement between aerial methods and the lysimeter. The Bowen ratio method yielded the best estimates of the lysimetric values. Comparison between the Bowen ratio method and the simplified aerodynamic method indicates a slight tendency for the last one to overestimate evapotranspiration when sensible heat fluxes are important (dry conditions, maturation).

It is shown that evapotranspiration may be predicted by means of a simple formula dependent upon net radiation, soil heat flux and air temperature as well as on a crop coefficient.

Bowen ratio method - simplified aerodynamic method - crop coefficient

\section{Introduction}

L'évapotranspiration (ET), encore appelée évapotranspiration réelle, est la perte en eau d'un couvert végétal sous forme vapeur par évaporation et transpiration pendant un intervalle de temps donné. Elle se distingue de l'évapotranspiration potentielle, obtenue lorsque toutes les surfaces d'échange (sol, végétation) sont saturées, de façon à ce qu'il n'y ait aucune restriction à l'eau disponible pour les plantes (Perrier, 1984).
La détermination de l'évapotranspiration avec une précision suffisante est un problème commun à ceux qui, à titres divers (agronomes, hydrologues, météorologistes), s'occupent des problèmes de gestion des ressources en eau, des liaisons entre consommation en eau et production végétale (Bouchet et Robelin, 1969; Puech et al., 1976) ou de la dépendance de ET à l'égard des facteurs climatiques et des caractéristiques de régulation des couverts végétaux (Katerji et Perrier, 1983). 
La quantification de l'évapotranspiration est possible par un grand nombre de méthodes : directement à l'aide d'un évapotranspiromètre, ou indirectement par le bilan hydrique ou les méthodes micrométéorologiques. Une revue complète de ces méthodes peut-être trouvée dans Mcllroy (1977) ou Sharma (1985). La comparaison de ces différentes méthodes a fait l'objet de plusieurs publications (Mukammal et al., 1966; Blad et Rosenberg, 1974). Toutefois, ces méthodes sont souvent comparées deux à deux, mais peu de travaux, tel Grant (1975), font état d'une comparaison simultanée de plusieurs d'entre elles.

L'objectif de cette étude, basée sur une expérimentation réalisée en Australie durant deux années sur blé non irrigué, est de comparer l'évapotranspiration obtenue de façon directe par un évapotranspiromètre, avec l'évapotranspiration calculée à partir des données de base publiées par Paltridge et al. (1972, 1975) en utilisant deux méthodes micrométéorologiques opérationnelles : 1) l'une classique : la méthode du rapport de Bowen; 2) l'autre proposée par Itier (1981) : la méthode aérodynamique simplifiée.

La comparaison simultanée de ces trois méthodes n'a pas encore été réalisée à notre connaissance.

Après un bref rappel théorique concernant les trois méthodes retenues, nous effectuons une comparaison des valeurs de ET à différentes échelles de temps (heure, jour et période) suivie d'une analyse critique de la précision et de la fiabilité des différentes méthodes.

Enfin, sur la base des données de ET obtenues, nous proposons une formule simple pour l'évaluation de l'évapotranspiration à partir d'une référence bioclimatique mesurable en routine.

\section{Présentation des méthodes de mesure de I'ET retenues}

\section{Méthode directe : l'évapotranspiromètre}

L'évapotranspiromètre pesable est une cuve contenant un volume de sol sur lequel est installé un couvert végétal de la façon la plus représentative possible par rapport au champ. En zone plane et hors irrigation ou pluie, la variation de poids du système est due à l'évapotranspiration (la variation due à l'augmentation de la masse végétale peut être considérée comme négligeable à l'échelle considérée). Différentes techniques de pesées ont été retenues : bascule mécanique, pesée piézométrique ou dynamométrique (Samie et de Villele, 1970).

Les sources d'erreurs expérimentales et la précision d'un évapotranspiromètre ont été analysées par Perrier et al. (1974). Ces auteurs ont noté un certain nombre de limitations et de contraintes liées à cette technique. Ils ont toutefois souligné que cette méthode reste indispensable pour vérifier ou tester les autres méthodes de mesure de l'évapotranspiration.

\section{Les méthodes micrométéorologiques retenues}

Les deux méthodes micrométéorologiques retenues dans le cadre de cette étude sont celles qui ont fait l'objet de recherches poussées en vue d'aboutir à des réalisations techniques. Il s'agit de la méthode du rapport de Bowen et de la méthode aérodynamique simplifiée. Les autres méthodes micrométéorologiques (aérodynamique combinée ou directe, fluctuations, thermométrie de surface) restent encore réservées aux laboratoires de recherche en raison d'une mise en $œ u v r e$ délicate.

La détermination de l'évapotranspiration par les deux méthodes envisagées ici repose sur l'équation du bilan d'énergie, valable dans la zone de flux conservatifs (couche limite de surface).

$L . E T=R n-G-H$

avec $R n$ : rayonnement net (W. $\mathrm{m}^{-2} ; \mathrm{G}$ : flux de chaleur par conduction à la surface du sol $\left(\mathrm{W} \cdot \mathrm{m}^{-2}\right) ; H$ : flux de chaleur sensible $\left(\mathrm{W} \cdot \mathrm{m}^{-2}\right) ; L$ : chaleur latente de vaporisation de l'eau $\left(2,46 \times 10^{6} \mathrm{~J} \cdot \mathrm{kg}^{-1}\right)$; $E T$ : évapotranspiration $\left(\mathrm{kg} / \mathrm{m}^{-2}\right.$ ou $\left.\mathrm{mm}\right) ;$ L.ET : flux de chaleur latente $\left(\mathrm{W} \cdot \mathrm{m}^{-2}\right)$.

Les flux sont pris positivement lorsqu'ils sont orientés vers le bas pour $R n$ et $G$ et vers le haut pour $H$ et L.ET.

Le principe commun à chacune des deux méthodes précipitées, consiste à mesurer les termes $R n$ et $G$ et à calculer le flux de chaleur sensible $H$ pour en déduire $E T$ à l'aide de l'équation 1. C'est dans le mode de détermination de $H$ que se distinguent les deux méthodes.

Méthode du rapport de Bowen. Cette méthode a été largement décrite dans la littérature. Elle consiste à déterminer le rapport $H / L$.ET à partir des mesures de différences de températures sèches et humides de l'air $(\Delta T$ et $\Delta T h$ ) à deux niveaux au dessus du couvert. En supposant l'égalité des diffusivités turbulentes pour les transferts de chaleur et de vapeur d'eau, L.ET est donné par l'expression suivante (Slatyer et Mcilroy, 1961):

$L . E T=(R n-G)\left(1-\frac{\gamma}{P^{\prime}(T h)+\gamma} \cdot \frac{\Delta T}{\Delta T h}\right)$

avec $\gamma$ : constante psychrométrique $\left(66 \mathrm{~Pa} \cdot \mathrm{K}^{-1}\right)$; $P^{\prime}(T h)$ : dérivée de la tension de vapeur saturante pour la température humide Th, calculée par la formule de Tetens (Murray, 1967).

Cette méthode a été à l'origine de la réalisation de deux systèmes de mesure (non utilisés ici) : le premier, analogique, a été mis au point par une équipe australienne (Mcllroy, 1971), le deuxième, numérique, appelé BEARN (bilan énergie automatique régional numérique) a été mis au point à I'INRA (Perrier et al. 1975). Ce dernier, en dehors de sa spécificité numérique, améliore le précédent par une inversion périodique des niveaux de mesure de $T$ et $T h$ afin d'augmenter la précision de mesure de $\Delta T h$, mesure délicate mais d'un poids déterminant dans cette méthode.

La méthode aérodynamique simplifiée. Cette méthode décrite par Itier (1981) constitue une simplification de la méthode aérodynamique combinée (Dyer \& Hicks, 1970; Webb, 1970). Le principe est de détermi- 
ner le flux de chaleur sensible $H$ à partir de mesures des gradients de vitesse du vent $(\Delta U)$ et de température $(\Delta T)$ entre deux niveaux au dessus du couvert végétal.

Le flux $H$ s'exprime dans la méthode aérodynamique simplifiée sous la forme : $H=f(\Delta U, \Delta T)$. La forme de la fonction $f$ dépend de la stabilité des basses couches atmosphériques. En fonction de $\Delta T$ ou $\Delta T /(\Delta U)^{2}$, on distingue 4 classes de stabilité.

L'organigramme de la Fig. 1 indique dans chaque cas l'expression de $H$. Dans le cas $n^{\circ} 3$, trois formules sont proposées pour $H$ : nous avons choisie celle de Riou (1981). Les paramètres $K, K r$, et $\alpha$ nécessaires pour le calcul (cf. Fig. 1), sont des fonctions simples de $z_{1}$ et $z_{2}$, hauteurs des niveaux de mesures respectivement bas et haut, prises depuis le sol et de $d$, hauteur de déplacement du plan de référence. Celle-ci est calculée à partir de la hauteur $h$ du couvert et de l'indice de surface foliaire total $L A \mid$ :

$d=h\left[1-\frac{2}{L A I}\left(1-\exp \left(-\frac{L A I}{2}\right)\right)\right]$ (Perrier, 1982)

La méthode aérodynamique simplifiée est à l'origine de la mise au point par l'INRA du système SAMER (système automatique de mesure de l'évapotranspiration réelle) qui présente l'avantage par rapport à la méthode de Bowen de s'affranchir de la mesure des températures humides.

Notons que cette méthode n'est applicable dans l'état actuel des choses que pour des niveaux de mesure grands devant la rugosité du couvert. Elle n'est plus valable au dessus de couverts développés tels que maïs ou canne à sucre (Cellier, 1984).

\section{Analyse d'erreur}

Méthodes du rapport de Bowen. Une analyse de l'erreur relative et absolue commise sur ET, liée à l'incertitude sur les paramètres mesurés, a été faite par Fuchs et Tanner (1970) ou Angus et Watts (1984). Notons simplement que, lorsque le gradient $\Delta T h$ est proche de zéro, les valeurs de $E T$ sont aberrantes.

Méthode aérodynamique simplifiée. De façon générale, l'erreur relative sur ET est particulièrement importante en début ou fin de journée, lorsque les gradients de températures sont faibles. Une analyse comparative de l'erreur liée à ces deux méthodes pourra être trouvée dans Peterschmitt (1987).

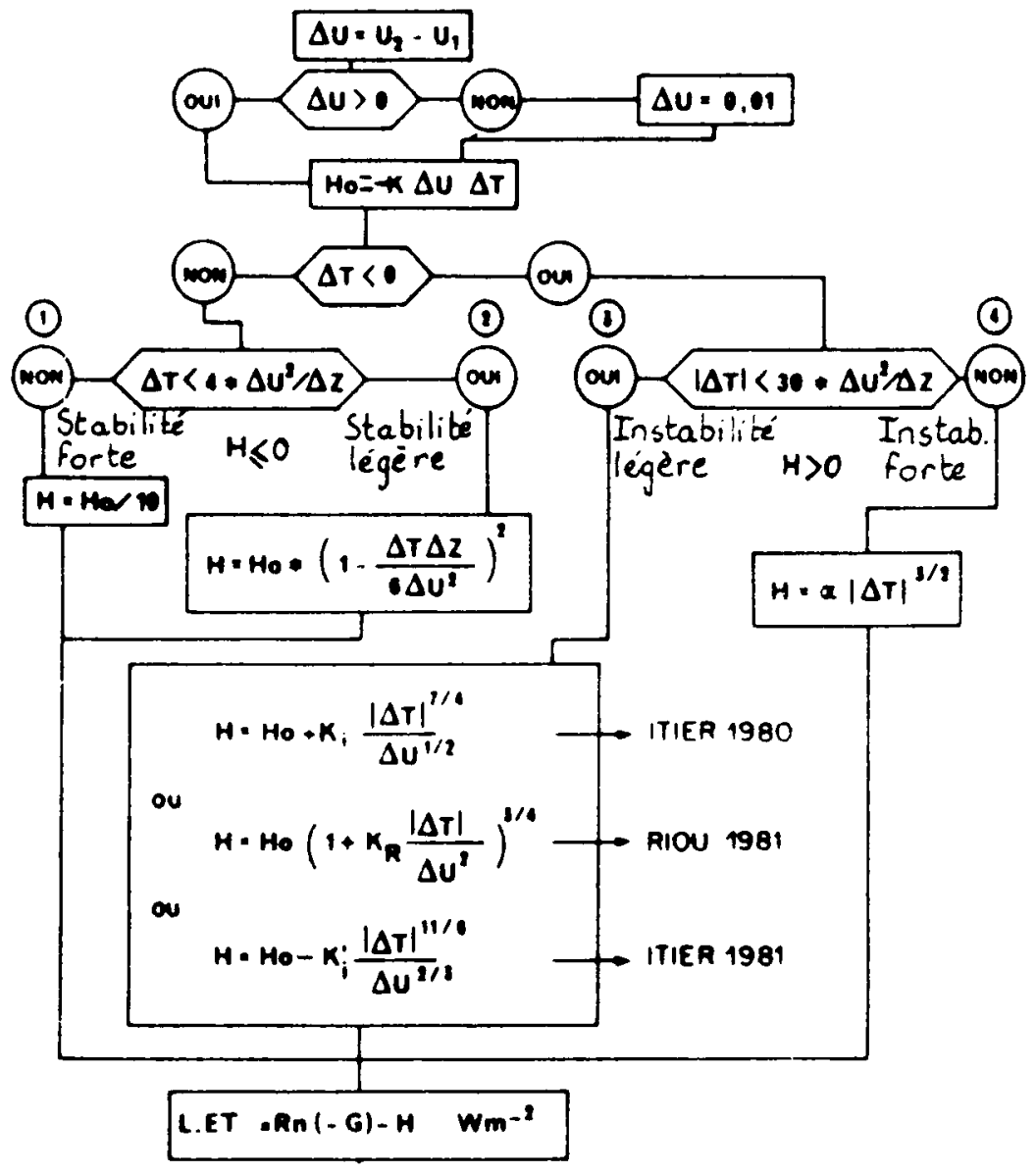

Fig. 1. Organigramme de calcul de l'évapotranspiration par la méthode aérodynamique simplifiée (d'après Itier, 1981). 


\section{Description de l'expérimentation}

L'ensemble des détails concernant le site expérimental et le matériel de mesure sont données par Paltridge et al. (1972).

\section{Le site}

L'expérimentation a été conduite à Rutherglen (Australie, $36^{\circ} \mathrm{S}-146^{\circ} \mathrm{E}$ ) en 1971 et 1972 sur blé non irrigué, variété Sherpa. La parcelle est de forme carrée, de dimensions $200 \times 200 \mathrm{~m}$ et entourée d'autres parcelles de blé sur une distance comprise entre 100 et $600 \mathrm{~m}$ selon les directions. Le sol est de texture argilosableuse et le climat de type océanique. La hauteur annuelle moyenne des précipitations est de $650 \mathrm{~mm}$, bien répartis dans l'année.

\section{Dispositif expérimental}

Mesure directe de ET. La mesure directe de ET est réalisée au moyen d'un évapotranspiromètre constitué par une cuve rectangulaire de dimensions $1.22 \times 1.52$ $\times 1.22 \mathrm{~m}$ en profondeur. La précision de la balance est de $\pm 0.05 \mathrm{~mm}$ en lame d'eau, soit environ $35 \mathrm{~W} \cdot \mathrm{m}^{-2}$ pour $1 \mathrm{~h}$.

Notons que durant la deuxième année, lors d'une période de dessèchement, les mesures par évapotranspiromètre ont été abandonnées, le blé installé sur la cuve présentant des signes de stress hydrique évident par rapport au champ.

Mesures micrométéorologiques. Les paramètres nécessaires pour le calcul de l'évapotranspiration par la méthode de Bowen ou la méthode aérodynamique simplifiée sont les suivants : $R n, G, \Delta U, \Delta T, \Delta T h, T$, Th.

Le rayonnement net et le flux de chaleur dans le sol sont mesurés respectivement à l'aide d'un pyrradiomètre différentiel placé à $2 \mathrm{~m}$ au dessus du sol et d'un fluxmètre placé à $2-3 \mathrm{~cm}$ sous la surface du sol. Les erreurs relatives commises par ces appareils sont respectivement de 2,5 et $5 \%$.

Les vitesses du vent sont mesurées à l'aide d'anémomètres sensibles à coupelles disposés à 1 et $2 \mathrm{~m}$ au dessus du couvert. La précision de la mesure, d'après Paltridge et al., 1972, est de $\pm 0,02 \mathrm{~m} \cdot \mathrm{s}^{-1}$ (seuil de démarrage $0,2 \mathrm{~m} \cdot \mathrm{s}^{-1}$ ).

Les gradients de températures sèche et humide sont mesurés à l'aide de sondes de platine placées sous abri ventilé à 1 et $2 \mathrm{~m}$ au dessus du couvert, avec inversion des niveaux bas et haut tous les quarts d'heure. La mesure est effectuée à l'aide d'un appareil à pont de Wheatstone. La précision annoncée (cf. Paltridge et al., 1972) est $\pm 0,01^{\circ} \mathrm{C}$, ce qui est difficile à réaliser pour $\Delta T h$.

Les températures absolues sèches et humides sont mesurées à l'aide de sondes de platine placés à $50 \mathrm{~cm}$ au-dessus du couvert.

L'ensemble de ces mesures est effectué en perrnanence, et des valeurs moyennes sont fournies à l'échelle horaire.

Deux fois par semaine, les hauteurs du couvert ainsi que l'indice de surface foliaire sont mesurés.

\section{Choix des journées}

Seule la période diurne durant laquelle $R n$ est supérieur à $50 \mathrm{~W} \cdot \mathrm{m}^{-2}$ a été prise en compte. Les premières et dernières heures de la journée ont été éliminées en raison des erreurs relatives trop importantes sur ET commises par les deux méthodes aériennes (voir ci-dessus).

Nous avons alors retenu les journées pour lesquelles l'ensemble des données étaient disponibles durant au moins $7 \mathrm{~h}$. Trois journées, présentant des valeurs aberrantes, ont été éliminées.

Finalement, l'analyse porte sur 74 journées, soit $572 \mathrm{~h}$, avec une bonne représentativité de l'ensemble des stades phénologiques, depuis la levée jusqu'à la maturation.

\section{Résultats}

Comparaison entre l'évapotranspiromètre et les méthodes aériennes

L.ET(eva) L.ET(bo) et L.ET(simp) désignent ici les flux de chaleur latente horaires mesurés en (W $\cdot \mathrm{m}^{-2}$ ) respectivement par l'évapotranspiromètre, les méthodes du rapport de Bowen et aérodynamique simplifiée. $E T($ eva), $E T(b o)$ et $E T(\operatorname{simp})$ désignent ici les évapotranspirations journalières (en $\mathrm{mm}$ ) mesurées par les trois méthodes en question.

\section{Valeurs horaires}

Les Figs. 2 et 3 présentent respectivement la comparaison entre L.ET(eva) et L.ET(bo) et entre L.ET(eva) et L.ET(simp). La première bissectrice et la droite de régression sont également représentées. On trouvera dans le Tableau I la pente et l'ordonnée à l'origine de la droite de régression (avec les écarts types), ainsi que le coefficient de corrélation élevé au carré.

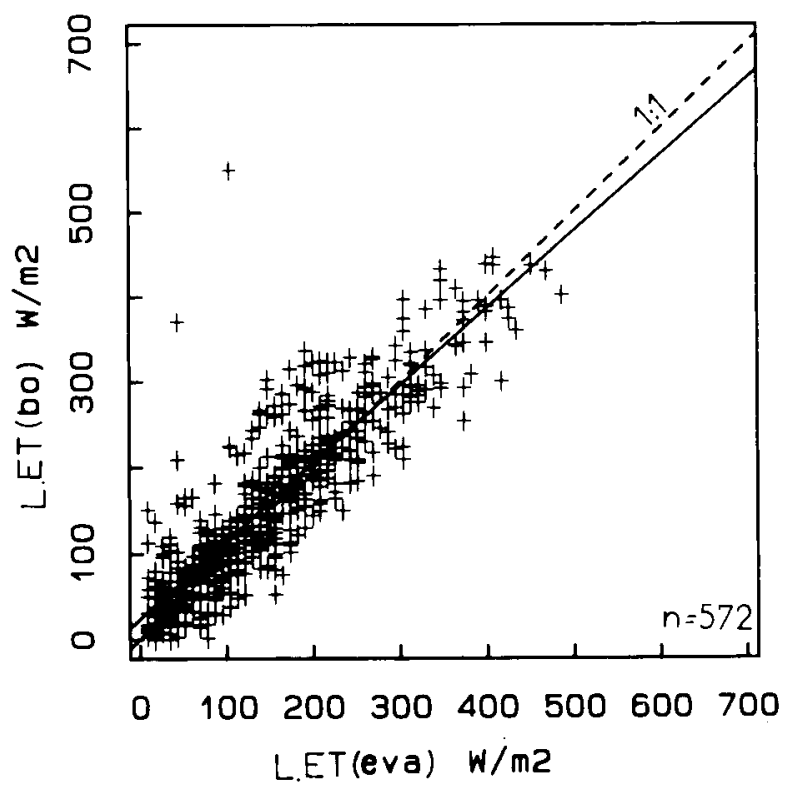

Fig. 2. Comparaison des valeurs horaires de flux de chaleur latente données par l'évapotranspiromètre L.ET(eva) et la méthode du rapport de Bowen L.ET (bo). 
Tableau I. Equations des droites de régression $Y=a X+b$, écarts types et coefficients de corrélation au carré pour les comparaisons entre les trois méthodes de mesure de l'évapotranspiration.

\begin{tabular}{|c|c|c|c|c|c|c|c|}
\hline Echelle & $x$ & $Y$ & $a$ & $\begin{array}{c}\text { Ecart type } \\
\quad a\end{array}$ & $b$ & $\begin{array}{c}\text { Ecart type } \\
b\end{array}$ & $\begin{array}{l}\text { Coefficient } \\
\text { de corrélation } \\
\text { au carré }\end{array}$ \\
\hline Horaire & L.ET (eva) & L.ET (bo) & 0,90 & 0,02 & 23,7 & 3,74 & 0,76 \\
\hline$\left(W \cdot m^{-2}\right)$ & L.ET (eva) & L.ET (simp) & 0,89 & 0,02 & 48,85 & 4,83 & 0,65 \\
\hline Jour & $E T$ (eva) & $E T$ (bo) & 0.95 & 0,04 & 0,17 & 0,09 & 0,86 \\
\hline$\left(m m \cdot j^{-1}\right)$ & $E T$ (eva) & $E T$ (simp) & 0,92 & 0,06 & 0,49 & 0,12 & 0,73 \\
\hline
\end{tabular}

L.ET(eva), ET(eva) : flux de chaleur latente horaire et évapotranspiration journalière, obtenus par l'évapotranspiromètre. L.ETbo), $E T(b o)$ : flux de chaleur latente horaire et évapotranspiration journalière, obtenus par la méthode du rapport de Bowen. $L . E T$ (simp) et $E T($ simp) : flux de chaleur latente horaire et évapotranspiration journalière, obtenus par la méthode aérodynamique simplifiée.

La correspondance entre les valeurs de L.ET(bo) et L.ET(eva) est satisfaisante. Par contre, la méthode aérodynamique simplifiée conduit à une dispersion plus importante, en particulier au-dessus de la bissectrice. Ceci suggère une erreur relative plus importante commise par cette méthode sur l'évapotranspiration.

\section{Valeurs journalières}

Les figs. 4 et 5 présentent comme précédemment les correspondances entre ET(eva) et $E T($ bo $)$ et entre $E T($ eva) et $E T($ simp). Comme cidessus, le Tableau I présente les coefficients de la régression. Les valeurs journalières sont obtenues à partir des cumuls horaires.

Nous constatons un bon accord général entre

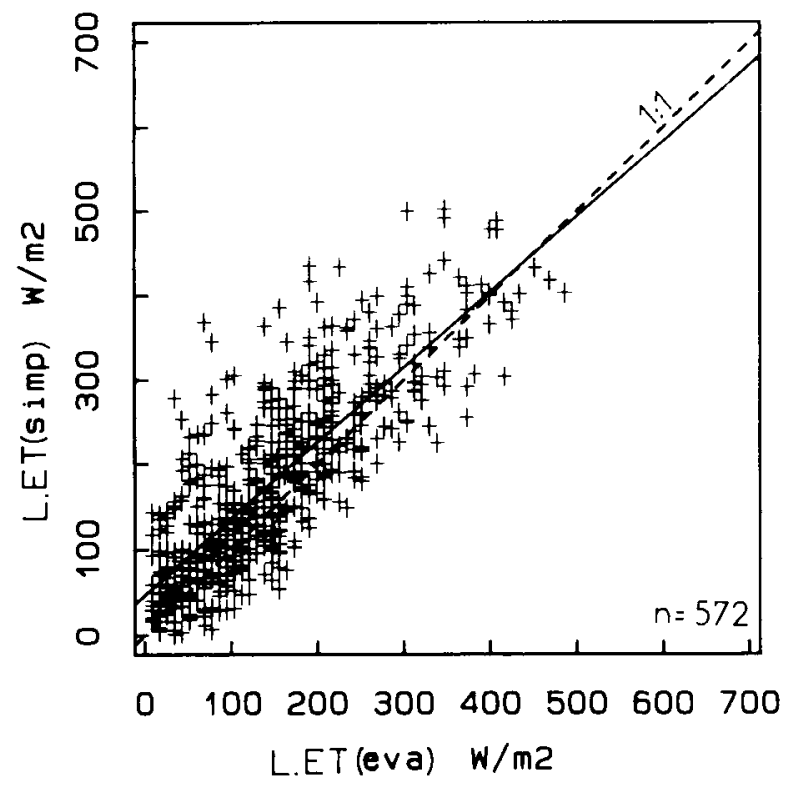

Fig. 3. Comparaison des valeurs horaires de flux de chaleur latente données par l'évapotranspiromètre $L . E T(e v a)$ et la méthode aérodynamique simplifiée L.ET(simp). les méthodes aériennes et l'évapotranspiromètre. Toutefois, pour un petit nombre de journées, les méthodes aériennes conduisent à des valeurs légèrement supérieures à $E T($ eva). Ceci est plus marqué pour la méthode aérodynamique simplifiée.

Les coefficients de corrélation obtenus sont meilleurs qu'à l'échelle horaire : une partie des différences observées s'annule donc par cumul.

Notons que Katerji et al. (1977), comparant la méthode de Bowen et l'évapotranspiromètre à l'échelle horaire et journalière sur maïs et blé, obtenaient des résultats très comparables à ceux-ci (équations de la droite de régression et coefficients de corrélation).

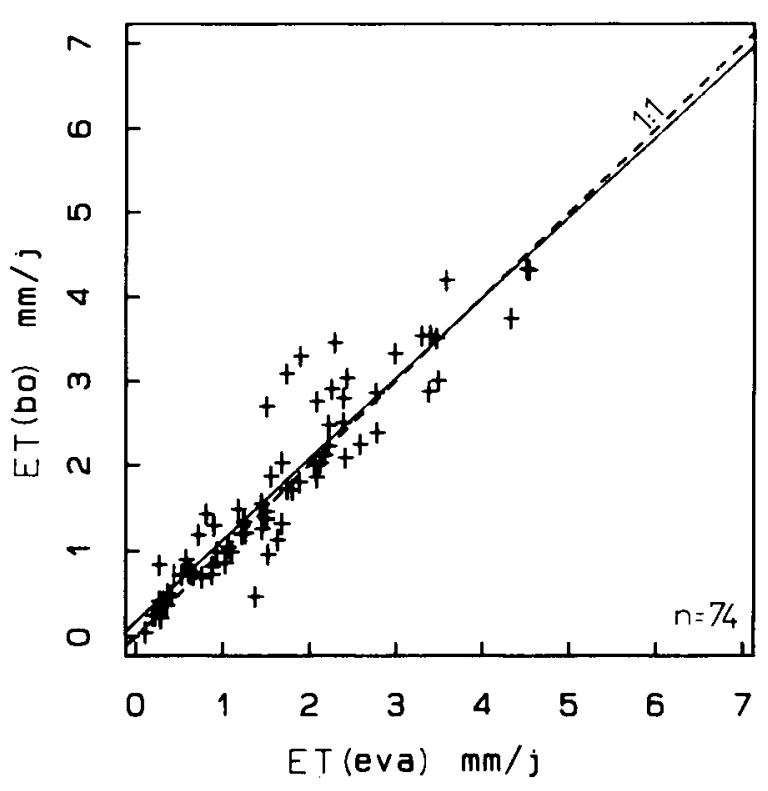

Fig. 4. Comparaison des valeurs journalières de l'évapotranspiration obtenues par l'évapotranspiromètre $E T(e v a)$ et la méthode du rapport de Bowen $E T(\mathrm{bo})$. 


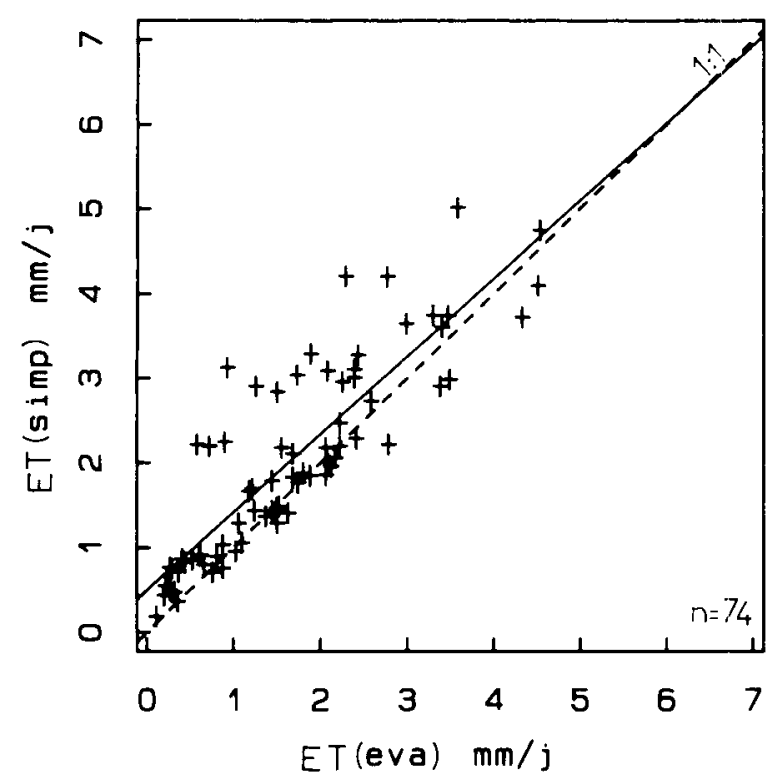

Fig. 5. Comparaison des valeurs journalières de l'évapotranspiration obtenues par l'évapotranspiromètre $E T($ eva) et la méthode aérodynamique simplifiée $E T($ simp).

\section{Valeurs cumulées}

La Fig. 6 présente l'évolution sur les 74 journées étudiées de l'évapotranspiration cumulée obtenue par les 3 méthodes pendant deux années successives (1971-1972).

Nous constatons l'exellent accord entre les valeurs cumulées de $E T$ obtenues par la méthode du rapport de Bowen et l'évapotranspiromètre, puisque la différence n'excède pas $6 \%$ à la fin de la période. Les valeurs cumulées de

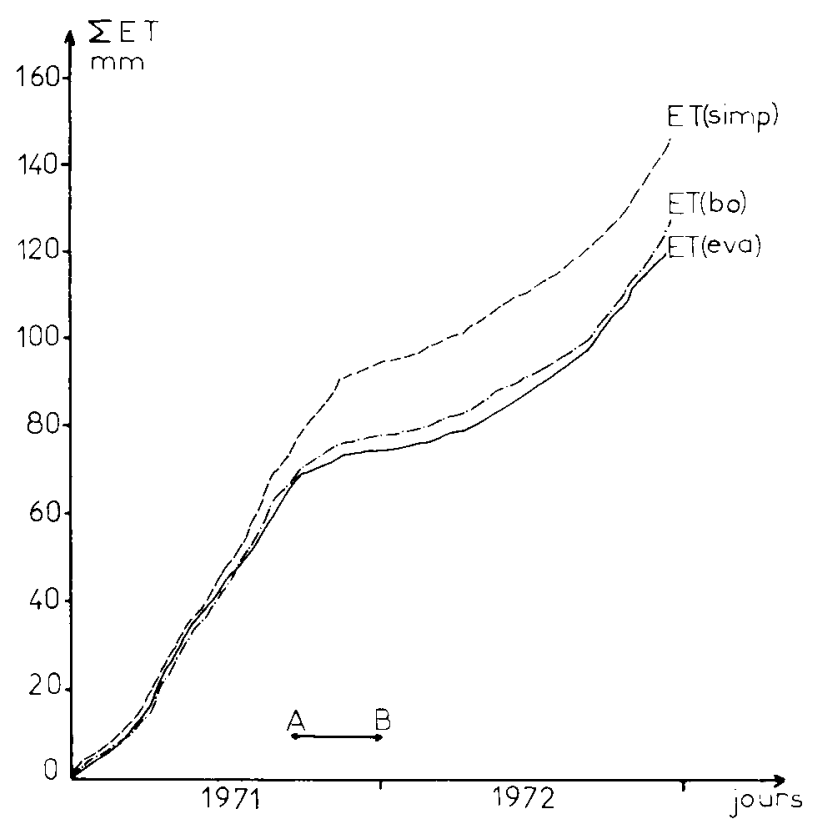

Fig. 6. Valeurs cumulées sur 74 jours des évapotranspirations obtenues par évapotranspiromètre $E T($ eva), la méthode du rapport de Bowen $E T($ bo) et la méthode aérodynamique simplifiée $E T$ (simp).
$E T$ obtenues par la méthode aérodynamique simplifiée présentent par contre un écart de $22 \%$ par rapport à celles obtenues par l'évapotranspiromètre.

L'analyse des courbes montre que cette différence est liée à un petit nombre de journées (période A-B sur la Fig. 6) situées à la fin du cycle cultural de 1971 et correspondant à la phase maturation, durant lesquelles la méthode aérodynamique simplifiée donne des valeurs supérieures à l'évapotranspiromètre.

\section{Comparaison des méthodes aériennes entre elles}

Pour la méthode de Bowen et la méthode aérodynamique simplifiée, la démarche a consisté à déterminer d'abord le flux de chaleur sensible $H$, puis à obtenir L.ET à partir du bilan d'énergie. II est donc préférable, pour la confrontation des résultats de comparer les valeurs de $H$ : en effet, selon le poids de $H$ par rapport à $R n-G$, l'erreur commise sur L.ET peut être inférieure à celle commise sur $H$.

Notons $H($ bo), et $H$ (simp) les flux de chaleur sensible horaire (en $\mathrm{W} \cdot \mathrm{m}^{-2}$ ) obtenus respectivement par les méthodes du rapport de Bowen et aérodynamique simplifiée. La Fig. 7 présente ces résultats et le Tableau II indique les coefficients de la régression entre les flux horaires de chaleur sensible et latente ainsi qu'entre les évapotranspirations journalières obtenues par les deux méthodes micrométéorologiques.

Nous constatons sur la Fig. 7 que la droite de

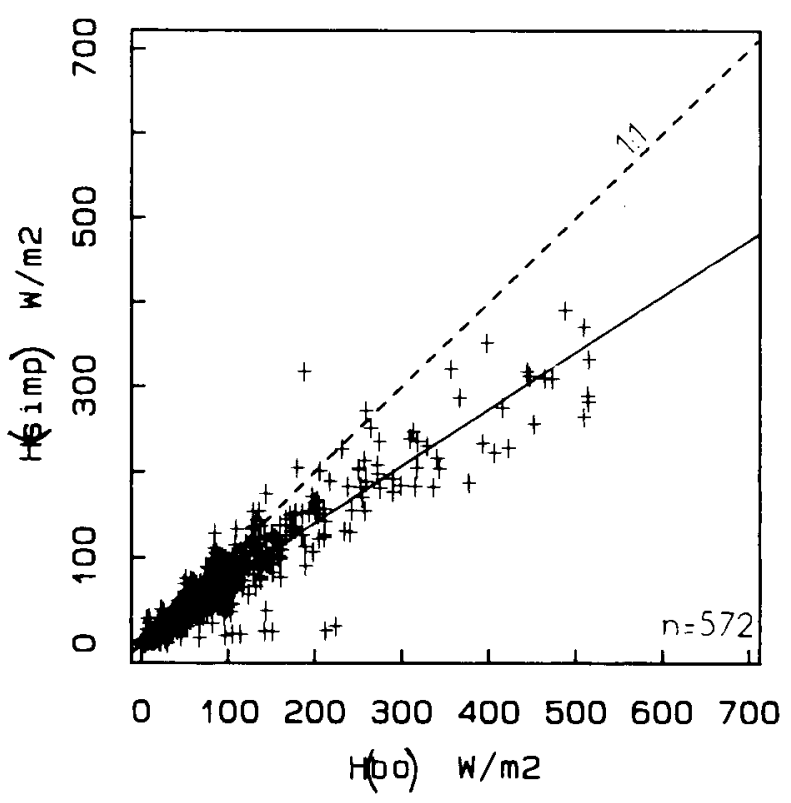

Fig. 7. Comparaison des flux de chaleurs sensibles horaires obtenus par les méthodes du rapport de Bowen $H(b o)$ et aérodynamique simplifiée $H$ (simp). 
Tableau II. Equations des droites de regression $Y=A X+b$, écarts types et coefficients de corrélation au carré pour les comparaisons entre les deux méthodes micro-météorologiques étudiées.

\begin{tabular}{llllllll}
\hline Echelle & $X$ & $Y$ & $a$ & $\begin{array}{c}\text { Ecart type } \\
a\end{array}$ & $b$ & $\begin{array}{c}\text { Ecart type } \\
b\end{array}$ & $\begin{array}{c}\text { Coefficient } \\
\text { de corrélation } \\
\text { au carré }\end{array}$ \\
\hline Horaire & $H(\mathrm{bo})$ & $H(\mathrm{simp})$ & 0,66 & 0,01 & 7,03 & 1,62 & 0,86 \\
$\left(\mathrm{~W} \cdot \mathrm{m}^{-2}\right)$ & L.ET(eva) & L.ET(simp) & 0,97 & 0,01 & 25,26 & 3,41 & 0,83 \\
Jour & ET (bo) & ET (simp) & 0,96 & 0,04 & 0,32 & 0,09 & 0,86 \\
$\left(\mathrm{~mm} \cdot j^{-1}\right)$ & & & & & & & \\
\hline
\end{tabular}

$H($ bo), $E T($ bo) : flux de chaleur sensible horaire et évapotranspiration journalière obtenus par la méthode du rapport de Bowen. $H$ (simp), ET(simp) : flux de chaleur sensible horaire et évapotranspiration journalière obtenus par la méthode aérodynamique simplifiée.

régression s'éloigne nettement de la bissectrice : les flux de chaleur sensible calculés par la méthode de Bowen sont supérieurs à ceux obtenus par la méthode aérodynamique simplifiée. Cette même tendance a également été mise en évidence par Saugier et Ripley (1978), sur prairie, en utilisant la méthode aérodynamique complète. Toutefois, ce phénomène est particulièrement marqué durant quelques journées, présentant des flux de chaleur sensibles importants et correspondant à la maturation. II en résulte une surestimation par la méthode aérodynamique simplifiée de l'évapotranspiration (cf. Fig. 6) par rapport à la méthode de Bowen (et, dans ce cas, à l'évapotranspiromètre).

Nous observons sur le Tableau II que, sur l'ensemble des journées, la différence relevée entre les flux de chaleur sensible donnés par les deux méthodes s'estompe lorsqu'on passe à l'évapotranspiration : la pente de la droite de régression est proche de 1.

Enfin, comme précédemment, nous constatons que les coefficients de corrélation sont plus élevés à l'échelle journalière qu'à l'échelle horaire.

\section{Une formule simple pour l'estimation de l'évapo-} transpiration

Katerji et Perrier (1983) ont proposé, pour exprimer l'évapotranspiration d'une culture, la formule suivante :

$$
E T=C \cdot\left[\frac{P^{\prime}(T a)}{P^{\prime}(T a)+\gamma}(R n-G)\right] / L
$$

D'après les auteurs, le coefficient $C$ assimilé à un coefficient cultural, dépend : 1) de la résistance globale opposée par le couvert au transfert de la vapeur d'eau; 2) des paramètres climatiques, maix ceux-ci n'entraînent pas de forte variation de $C$; 3) du stade phénologique, $C$ diminuant nettement au cours de la maturation; 4) des conditions hydriques du sol, $C$ diminuant en situation de dessèchement.

Dans le cas présent, nous avons choisi d'estimer $C$ à partir des valeurs d'évapotranspiration fournies par la méthode de Bowen. La Fig. 8 présente la relation entre les valeurs journalières de $E T($ bo $)$ et les valeurs correspondantes du terme $\left[\left(P^{\prime} /\left(P^{\prime}+\gamma\right)(R n-G)\right] / L\right.$.

Le Tableau III présente les valeurs de $C$ obtenues (en imposant une ordonnée à l'origine nulle) pour deux groupes distincts de journées : les journées durant le stade vert (ensemble du cycle cultural sauf maturation) et les journées correspondant à la maturation. Dans chaque cas, les écarts-types et les coefficients de corrélation sont mentionnés.

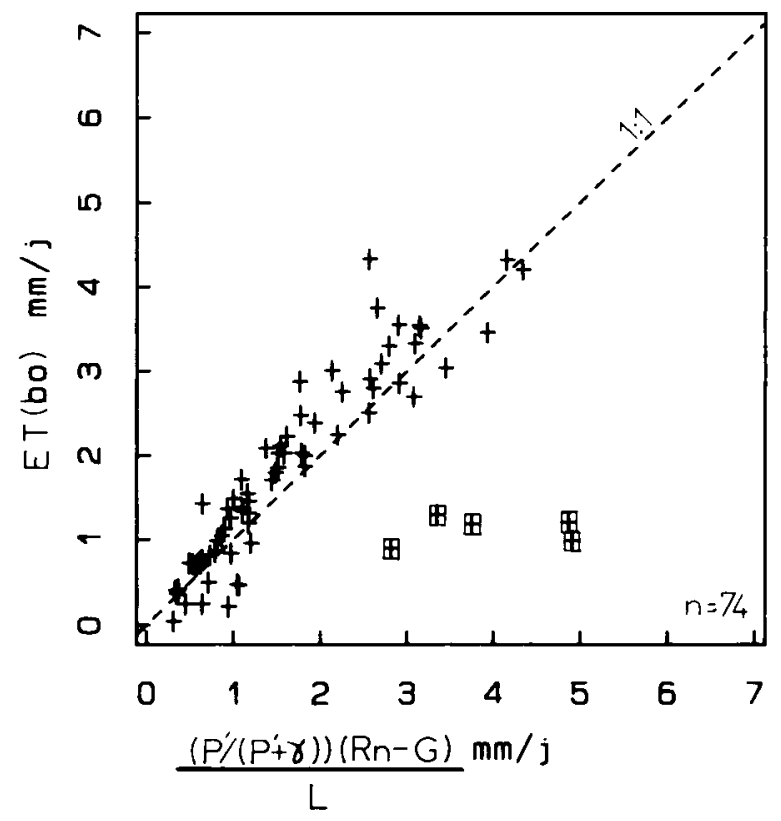

Fig. 8. Comparaison des valeurs journalières de l'évapotranspiration obtenues par la méthode du rapport de Bowen $E T($ bo $)$ et le terme $\left[\left(P^{\prime} /\left(P^{\prime}+\gamma\right)\right) \cdot(R n-G)\right] / L$. 
Tableau III. Valeurs du coefficient $C$, écarts types et coefficients de corrélation au carré pour le stade vert et la maturation.

\begin{tabular}{llll}
\hline & $c$ & $\begin{array}{c}\text { Ecart type } \\
C\end{array}$ & $\begin{array}{l}\text { Coefficient de } \\
\text { correlation au carré }\end{array}$ \\
\hline $\begin{array}{c}\text { Totalité des journées sauf } \\
\text { maturation (stade vert) }\end{array}$ & 1,11 & 0,02 & 0,96 \\
\begin{tabular}{l} 
Maturation \\
\hline
\end{tabular} & 0,27 & 0,03 & 0,94 \\
\hline
\end{tabular}

Les journées du stade maturation se détachent nettement de l'ensemble des points sur la Fig. 8 (points encadrés). Le coefficient de corrélation obtenu pour le restant du cycle (stade vert) est très élevé, indiquant la bonne correspondance entre la formule simplifiée et les valeurs données par la méthode du rapport de Bowen.

Notons enfin que la valeur de $C$ observée durant le stade vert est très proche de celle obtenue également sur blé par d'autres auteurs : Katerji et al., (1977) dans le bassin parisien, Denmead \& Mcllroy (1970) en Australie ou Hanks et al., (1968) dans les plaines centrales des EtatsUnis (voir revue bibliographique de Katerji et Perrier, 1983).

\section{Discussion-Conclusion}

La comparaison de ces trois méthodes suggère plusieurs réflexions.

Nous avons constaté que les méthodes micrométéorologiques étudiées conduisent dans l'ensemble à un bon accord avec l'évapotranspiromètre. La méthode de Bowen est celle qui fournit les valeurs les plus proches de l'évapotranspiromètre. Par rapport à l'évapotranspiromètre, lourd et immobile, les méthodes aériennes présentent plusieurs avantages. Elles constituent des systèmes légers et mobiles. De plus, ces méthodes apparaissent plus représentatives du champ que l'évapotranspiromètre : d'une part le milieu n'est pas perturbé, d'autre part, les gradients de température ou de vitesse du vent résultent d'une intégration des flux sur une surface plus grande que celle de l'évapotranspiromètre.

Toutefois, une bonne précision des résultats nécessite un strict suivi technique.

Notons enfin que, lors de situations de dessèchement important, l'évapotranspiromètre peut ne plus être représentatif du champ, comme le soulignent Paltridge et al. (1975) durant la deuxième année de mesure. D'une part, la profondeur d'enracinement au champ peut être supérieure à la profondeur de la cuve, d'autre part cette dernière constitue un milieu fermé ne prenant pas en compte les remontées capillaires. Celles-ci peuvent représenter une part importante de l'évapotranspiration (Katerji et al., 1977).

La comparaison des méthodes aériennes entre elles montre que la méthode aérodynamique simplifiée à tendance à sous-estimer le flux de chaleur sensible et donc à surestimer $E T$ lorsque les flux de chaleur sensible sont élevés (cas de la maturation ou d'un déficit hydrique important).

Néanmoins, la méthode aérodynamique simplifiée reste d'un emploi plus facile que la méthode du rapport de Bowen : la mesure délicate des températures humides est évitée et il n'est pas nécessaire d'envisager l'inversion automatique des niveaux bas et haut des capteurs de température.

Enfin, d'un point de vue pratique, l'utilisation d'une formule simple, basée sur la connaissance d'un coefficient $C$ et d'une mesure en routine de $R n, G$ et $T$ conduit à de bonnes estimations de l'évapotranspiration d'une culture lorsque les conditions hydriques du sol ne sont pas limitantes et les mesures non perturbées par des apports d'énergie d'origine advective. Le degré de précision obtenu est tout à fait suffisant pour des objectifs tels que la conduite de l'irrigation.

\section{Références}

Angus D.E. \& Watts P.J. (1984) Evapotranspiration. How good is the Bowen ratio method? Agric. Water Manage. 8, 133-150

Blad B.L. \& Rosenberg N.J. (1974) Lysimetric calibration of the Bowen ratio-energy balance method for evapotranspiration estimation in the Central Great Plains. J. Appl. Meteorol. 13, 227-236

Bouchet R.J. \& Robelin M. (1969) Evapotranspiration potentielle et réelle. Domaine d'utilisation. Portée pratique. Bull. Tech. Inf., 238, 215-223

Cellier P. (1984) Tentative de correction des relations flux-gradients au voisinage de surfaces très rugueuses. J. Rech. Atmos. 18, 253-262

Denmead O.T. \& Mcllroy I.C. (1970) Measurements of non-potential evaporation from wheat. Agric. Meteorol. 7, 285-302 
Dyer A.J. \& Hicks B.B. (1970) Flux-gradient relationships in the constant flux layer. Q.J. R. Meteorol. Soc. 96, 715-721

Fuchs M. \& Tanner C.B. (1970) Error analysis of Bowen ratios measured by differential psychrometry. Agric. Meteorol. 7, 329-334

Grant D.R. (1975) Comparison of evaporation measurments using different methods. Q.J.R. Meteorol. Soc. 101, 543-550

Hanks R.J., Gardner H.R. \& Cury E.E. (1968) Evapotranspiration climate relation for several crops in the Central Great Plains. Agron. J. 5, 538-542

Itier B. (1981) Une méthode simple pour la mesure de l'évapotranspiration réelle à l'échelle de la parcelle. Agronomie 1, 869-876

Katerji N., Gosse G., Perrier A. \& Daudet F.A. (1977) Etude suivie de l'évapotranspiration réelle d'un couver de blé et de maïs au moyen d'un dispositif automatique B.E.A.R.N. La Météorologie, V|e série, 11, 45-53

Katerji N. \& Perrier A. (1983) Modélisation de l'évapotranspiration réelle ETR d'une parcelle de luzerne : rôle d'un coefficient cultural. Agronomie 3, 513-521

Mcllroy I.C. (1971) An instrument for continuous recording of natural evaporation. Agric. Meteorol. 9, 93-100

Mcllroy I.C. (1977) A systematic review of practical evaporation determination in the field. Conf. Int. sur l'Evapotranspiration, C.I.I.D., Budapest mai 1977, Abst. p. 21

Murray F.W. (1967) On the computation of saturation vapor pressure. J. Appl. Meteorol. 12, 1410-1411

Mukammal E.l., King K.M. \& Cork H.F. (1966) Comparison of aerodynamic and energy budget techniques in estimating evapotranspiration from a cornfield. Arch. Meteorol. Geophys. Bioklimsatol. Ser. B., 14, 384-395

Paltridge G.W., Dilley A.C., Garrat J.R., Pearman G.l., Sheperd W. \& Connor D.J. (1972) The Rutherglen experiment on Sherpa wheat: environmental and biological data. Division of Atmospheric Physics: technical paper no. 22, Commonwealth Scientific and Industrial Research Organization, Australia

Paltridge G.W., Dilley A.C., Sheperd W. \& Connor D.J. (1975) The Rutherglen experiment on Sherpa wheat: environmental and biological data. II. Division of Atmospheric Physics: technical paper no. 26, Commonwealth Scintific and Industrial Research Organization, Australia

Perrier A. (1982) Land surface processes: vegetation. In: Land surface processes in atmosphere general circulation models, World Climate Research Programme study conference, WMO/ICSO, Greenbelt, MD,
(Eagleson P.S., Ed.), USA, Cambridge University Press, Cambridge, pp. 395-448

Perrier A. (1984) Mise à jour des définitions sur l'évapotranspiration et les besoins en eau des cultures. In: Les besoins en eau des cultures - Crop water requirements (Perrier A. \& Riou C., eds.), Conférence Internationale, Commission Internationale des Irrigations et du Drainage, Paris, INRA, Paris, pp. 885-887

Perrier A., Archer P. \& Blanco de Pablos A. (1974) Etude de l'évapotranspiration réelle et maximale de diverses cultures: dispositif et mesures. Ann. Agron. $25,697-731$

Perrier A., Itier B., Bertolini J.M. \& Blanco de Pablos A. (1975) Mesure automatique du bilan d'énergie d'une culture. Exemples d'application. Ann. Agron. 26, 1940

Peterschmitt J.M. (1987) Comparaison de différentes méthodes de mesure de l'évapotranspiration d'un couvert de blé non irrigué (Rutherglen - Australie). Mémoire de D.E.A., ENGREF Montpellier, INA-PG, INRA Bioclimatologie Grignon

Puech J., Marty J.R. \& Maertens E. (1976) Efficience de l'eau consommée par divers végétaux et application à la valorisation de l'irrigation. Bull. Tech. Inf., 306, 41-53

Riou C. (1981) Une nouvelle méthode de détermination du flux de chaleur convective à partir de mesures de température et de vitesse du vent à deux niveaux. Son application à la mesure de l'évaporation et sa mise en œuvre en Tunisie. I - Bases théoriques et mise en œuvre. Cahiers ORSTOM Sér. Hydrol. XVIII, 3-9

Samie B. \& de Villèle O. (1970) Méthodes et techniques de mesure de l'évaporation et de l'évapotranspiration In: Techniques d'étude des facteurs physiques de la biosphère (Hallaire M. et al., ed.), Paris, pp 257-266

Saugier B. \& Ripley E.A. (1978) Evaluation of the aerodynamic method of determining fluxes over natural grassland. Q. J. R. Meteorol. Soc. 104, 257-270

Sharma M.L. (1985) Estimating evapotranspiration. in: Advances in irrigation, (Hillel D., ed.), Vol. 3, Academic Press, London, pp. 214-281

Slatyer R.O. \& Mcliroy I.C. (1961) Evaporation and the principle of its measurment. In: Pratical microclimatology (Slatyer R.O. \& Mcllroy I.C., eds.), CSIRO-UNESCO, pp. 50-60

Webb E.K. (1970) Profile relationships: the loglinear range, and extension to strong stability. $Q$. J. $R$. Meteorol. Soc. 96, 67-90 